\title{
Zur biologischen Bedeutung der Kreuzung der Leitungsbahnen im Zentralnervensystem.
}

\author{
Von \\ Kingo Kato. \\ (Aus dem Anatomischen Institut der Medzinischen Fakultät zu Niigata, \\ Japan. Leiter: Prof. Dr. Ko Hirasawa.)
}

Über die Entstehungsweise der Kreuzung der Leitungsbahnen im Zentralnervensystem existiert schon eine Reihe von Arbeiten wie von Flechsig (1876), Cajal (1898), Wundt (1908), Spitzer (1910), Jakobsohn-Lask (1924 u. 1928), Miskolczy (1926) u. a. m, wemn auch keine der von ihnen gegebenen Deutungen allgemeine Anerkennung gefunden hat. Üher die biologische Bedeutung derselhen jedoch, soweit mir bekannt, gibt es noch fast keine Literatur. In der vorliegenden Abhandlung will ich daher die biologische Bedeutung der Leitungsbahnen zu erklären rersuchen. Bevor ich anf diese Frage eingehe, will ich zunächst auf das Schrifttum über den Entstehungsmechanismus der Kreuzung der Leitungsbahnen einen kurzen Blick werfen, da dieser zu der Frage, womit ich mich hier beschäftige, in enger Beziehung steht.

Flechsig schrieb die Kreuzung der Pyramidenbahn einem gewissen Widerstande, der sich nach ihm während der ontogenetischen Entwicklung an der Knickungsstelle zwischen dem Kopf- und Rückenmark zeigen sollte, zu.

Den triftigen, schwerwiegenden Argumenten von Spitzer und Jakobsohn-Lask, wonach mit Hilfe solcher Annahmen höchstens nur das Verhalten der Pyramidenbahn und deren Kreuzungsvariabilität erklärbar sei und einem so nebensächlichen Momente keine entscheidende Gültigkeit zugeschrieben werden könne, hat Miskolczy mit Recht hinzugefügt, dass es ziemlich gewagt sei, sich bei der normalen Entwicklung auf Behind- 
erungen $\mathrm{zu}$ berufen, welche während des Entwicklungsvorganges bei jedem Fall neu zu überwinden wären.

Nach Ramon y Cajal soll sich die Kreuzung infolge der überragenden Herrschaft des Sehorgans bei den niederen Tieren zuerst an den Sehfasern vollziehen und zwar deshalb, weil sich das panoramische Sehfeld bei homolateralem Verlauf der Sehfasern infolge der Linsenbrechung in zerteilter und ungekehrter Gestalt auf die Netzhaut und von dieser auf die Hirnrinde projizieren und das Bewustsein also ein verkehrtes Bild aufnehmen würde. Dieser führenden Kreuzung hätten sich dann im Laufe der phylogenetischen Entwicklung sämtliche andere Nervenbahnen angepasst.

Gegen diese Theorie hat Jakobsohn-Lask ganz triftig eine Reihe von Bedenken erhoben :

,, Einmal hätte Cajal die Verhältnisse bei den Wirbellosen nicht berücksichtigt bzw. zu gering eingeschätzt. Zweitens wäre bei den niederen Tieren das Sehorgan nicht das alle anderen Sinne so überragende, dass es im gesamten Lebensprozess die Führung habe, sondern das Riechorgan sei wohl das zunächst führende, stehe jedenfalls an Bedeutung dem Sehorgan nicht nach. Drittens sei es eine etwas naive Anschauung, dass das Bewusstsein das der Rinde zugeleitete Bild einfach wie von einer photographischen Platte ablese, vielmehr werden die Selseindrücke in der Hirnrinde durch einen in seinen Einzelheiten ganz unbekannten Vorgang verarbeitet, und das vielleicht durch die Sehmedien verkehrt projizierte Bild werde wieder vom Bewusstsein nach aussen projiziert und in dieser nach aussen, also richtig projizierten Form im Bewusstsein erhalten, ganz ebenso wie es mit allen Eindrücken geschähe, die den tierischen Körper von aussen treffen. Viertens müssten ja, wenn die Theorie von Cajal zuträfe, sich die Sehfaseru nicht nur in der horizontalen, sondern auch in der senkrechten Ebene kreuzen, was doch nicht der Fall sei. Fünftens ist es eine willkürliche Annahme, dass eine an einer lokalen Stelle eingetretene Kreuzung nun sämtliche Bahnen des Zentralnervensystems in gleicher Weise beeinflusst haben sollte, vielmehr ist das Kreuzungsphänomen ein allgemeines, welches auch nur eine allgerneine Ursache haben könne. Aus diesen und noch anderen Gründen hat die Theorie von Cajal, so bestechend sie auch auf den ersten Blick erscheint, keine Annahme gefunden."

Wundt war zuerst der Ansicht, dass die Kreuzungen der Leitungsbahnen im Zentralnervensystem der Säugetiere deshalb entstanden seien, um zwischen der rechten Körperhälfte einerseits, welche die Säugetiere in ihrem Lebenskampfe instinktmässig zur Schützung des links gelagerten 
Herzens in stärkerem Masse als die linke Körperhälfte benutzen und welche dadurch kräftiger als diese ausgebildet sei und der linken Hemisphäre anderseits, welche durch angeblich reichlichere Blutversorgung infolge der Linkslagerung des Herzens zu einem Übergewicht gelangt wäre, einen gewissen Ausgleich zustandezubringen. Diese Hypothese liess er aber selbst später fallen und schloss sich wenigstens in grossen Zügen, der oben angeführten Cajal'schen Anschauung an.

Nach A. Spitzer, welcher den Körper der Wirbeltiere von dem der Wirbellosen abzuleiten versuchte, wandelt sich ein Teil des Darmkanals und zwar nicht der ursprünglich dorsal, sondern der ventral gelegene Teil desselben der Wirbellosen im Laufe der phylogenetischen Entwicklung zum Zentralkanal um, indem der ursprünglich ventral gelegene Teil des Darmkanals durch eine Drehung des Körpers in der Längsachse urn $180 \mathrm{Grad}$ von ventral nach dorsal und der ursprünglich dorsale Teil ungekehrt, nämlich von dorsal nach ventral gerückt wäre. Diese Torsion der zwei Kanalabschnitte liefere gerade an dem Drehpunkt, welcher am vorderen Pol der Chorda dorsalis in der Infundibulargegend gelegen haben soll, die Grundlage der ersten totalen Kreuzung der Leitungsbahnen, welche dann alle weiteren Kreuzungen nach sich gezogen hätte. Der vor diesem Drehpunkt befindliche Körperteil sei in seiner ursprünglichen Lage unverändert geblieben, bätte sich also nicht gedreht.

Gegen diese Hypothese von Spitzer hat Jakobsohn-Lask eine Reihe von Einwänden erhoben. Er sagt nämlich, dass sie auf einer ganz künstlichen Konstruktion beruhe, indem sich für die Drehung des Körpers weder in der Onto- noch in der Phylogenese ein sicherer Anhaltspunkt finde, und ferner, dass, selbst wenn man dem Autor die Möglichkeit eines solchen Vorganges konzediert, die Kreuzungen im vorderen Abschnitt der Neuralachse, die sich ja nach Spitzers Ammahme nicht gedreht hat, demmoch sich nicht erklären liessen.

Besonders stark betont Jakobsohn-Lask, dass man bei der Untersuchung eines solchen Problems wie das über die Kreuzung der Leitungsbahnen, die nach ihm eine allgemeine fundamentale Erscheinung im Tierkörper ist, auch die Verhältnisse bei Wirbellosen unbedingt mit in Rücksicht nehmen müsse.

In einer kurzen Abhandlung hat Miskolczy über Bedeutung und Notwendigkeit der Kreuzungen gesprochen. Nach ihm sei die Endursache der Bilateralität des Nervensystems und des Grundskeletts in der Lokomotion zu suchen, welche das Besorgen der Nahrung erfordert, und das Ausführen alternierender, synergetischer bezw. antagonistischer Funktionen mache es notwendig, dass die entsprechenden Bewegungs- 
zentren des Nervensystems nicht nur mit den exekutiven Organen der gleichen, sondern auch der gegenüberliegenden Seite in konstanter Verbindung seien.

Jakobsohn-Lask versuchte wie Spitzer die Kreuzung der Bahnen im Zentralnervensystem der Wirbeltiere von denen der Wirbellosen abzuleiten.

Bezüglich der Entstehung der Nervenbahmen und der Kreuzung derselben bei den Wirbellosen behauptet er, dass das Nervensystem der niedersten Wirbellosen, d.h. die einfachste Form des Nervensystems, ein in gleichmässiger Art in der Wand des Ektodermalsackes gelegenes, diffuses Nervennetz bilde.

Auf der zunächst höheren Tierstufe (Raupen, Spinnen und viele andere Tiere) konzentriere sich ein Teil von diesem diffusen Nervennetz und verlagere sich von der Körperperipherie in das Körperinnere. In diesem konzentrierten Teil entwickeln sich aus dem Netz mehr selbständige Bahnen. Da nun diese sich isolierenden Bahnen aus dem ursprünglichen Netze hervorgehen und dieses Netz in seiner Totalität einen Nervenzylinder bildet, der wiederum seinerseits im Körperzylinder gelegen sei, so wäre es einleuchtend, dass der Verlauf der sich isolierenden Fasern ein teils gekreuzter, teils ungekreuzter sein müsse. Die Ansicht Cajals, dass die zuerst aufgetretene Kreuzung eine totale gewesen sei, und dass sich erst später aus dieser totalen Kreuzung die partielle herausgebildet hätte, hält Jakobsohn-Lask daher für nicht richtig. Vielmehr sei nach diesem Autor die partielle Kreuzung der Nervenbahnen in der tierischen Entwicklung das Primäre gewesen und die totale habe sich an viel wenigeren Stellen aus besonderen Umständen herausgebildet.

Über die Kreuzung der Bahnen im Zentralnervensystem der Wirbeltiere führt er an :

, Nach den Ansichten sehr vieler hochbedeutender Forscher soll sich nun das Nervensystem der Wirbeltiere aus dem der Wirbellosen entwickelt haben.... Man muss aber doch wohl annehmen, dass auch das Zentralnervensystem der Wirbeltiere aus einfachsten Verhältnissen entstanden ist, dass die Grundlage des Nervensystems auch bier ein Nervemnetz gewesen ist, aus dem nach und nach immer mehr isolierende Bahnen sich herauskristallisiert haben. So weit wir es jetzt noch beobachten können, entsteht das Zentralnervensystem der Wirbeltiere aus einer kondensierten Sinnesplatte des Ektoderms, die sich sehr schnell zu einem Hohlzylinder umformt, und aus der Wand dieses Nervenhohlzylinder's (Medullarrohr), der sich auch allmählich ins Imnere des Körperzylinders verlagert, entwickeln sich die Nervenzellen, das Nervenfaser- 
grundnetz bzw.-geflecht, und die isoliert verlaufenden Nervenbahnen. Da nun sowohl der Körper der Wirbeltiere als auch das Zentralnervensystem, jedes für sich, einen Hohlzylinder bilden, und da auch sämtliche Körperanhänge (Extremitäten), sowie die inneren Organe sich dieser Gestaltform anpassen, d.h. reifenförmig ihn umfassen oder in ihm liegen, so muss auch hier der Nervenstrom immer kreisförınig durch die Nervenbahnen des Körpers und durch diejenigen des Zentralnervensystems hin- und zurückfluten, und so ist es auch hier ganz natürlich, dass sich die Nervenbahnen im Zentralnervensystem der Wirbeltiere teils kreuzen, teils ungekreuzt verlaufen, und dass auch hier, ebenso wie bei den Wirbellosen, nicht nur lockere einzelne Fasern vorhanden sind, sondern daneben zahllose ganz besonders starke Systeme entstanden sind, die sich im Laufe der Entwicklung aus Fasern von gleicher Funktion zusammengeballt haben."

Dieser Erklärungsversuch von Jakobsohn-Lask für die Kreuzung der Nervenbahnen ist gewiss von allen bis jetzt veröffentlichten Hypothesen die begründetste, aber noch nicht ganz frei von Schwächen und gegen sie lassen sich noch manche Einwände erheben.

Erstens muss es noch späteren Forschungen vorbehalten bleiben, ob die Wirbeltiere wirklich von den Wirbellosen direkt abstammen oder ob noch andere Möglichkeiten vorliegen. Gegenwärtig ist jeder speziellere Ableitungsversuch der Wirbeltiere von den Wirbellosen, wie Kuhlenbeck betont, nicht mehr als eine auf sehr unsicheren Grundlagen beruhende theoretische Konstruktion. Auf die einzelnen diesbezüglichen Hypothesen kann hier nicht näher eingegangen werden, weil dies zu weit führen würde.

Zweitens sagt Jakobsohn-Lask, dass sich die Nervenbahnen im Zentralnervensystem deshalb teils kreuzen, weil sie aus dem ursprünglichen diffusen Netz hervorgegangen seien. Wenn auch diese Behauptung auf den ersten Blick so ,, einleuchtend“ wirkt, so merkt man aber bei etwas näherer Betrachtung sofort, dass die gegebene Argumentation nicht hinreichend ist, die Kreuzung der Nervenbahnen zu erklären.

Wenn man auch dem Autor zugibt, dass sich die Nervenbahnen kreuzen, weil sie ursprünglich aus einem diffusen Netz hervorgegangen seien, so kann hiermit noch nicht dargelegt werden, warum die Kreuzung der Nervenbahnen, welche aus mehr als zwei Neuronen zusammengesetzt sind, immer regelmässig zu bestimmten Malen an bestimmten Stellen stattfindet. Die Pyramidenbahnen setzen sich bekanntlich aus zwei Neuronen, von welchen das erste den motorischen Zellen des motorischen Rindenfeldes und deren Achsenzylindern und das zweite 
denen der motorischen Hirnnervenkerne oder des Rückenmarksvorderhorues entspricht, zusammen. Sie könnten sich also wenigstens zweimal kreuzen, vorausgesetzt, dass sich jedes Neuron nur einmal kreuzt, aber in Wirklichkeit nur einmal und zwar nur am ersten Neuron. Die Leitungsbahnen der Hautsensibilität wie der Tractus spinothalamicus, bulbothalamicus etc. setzen sich aus drei Neuronen zusammen und deshalb könnten sie sich ceteris paribus dreimal kreuzen, aber in der Tat kreuzen sie sich alle nur einmal und zwar stets am zweiten Neurone.

Meines Erachtens könnte wohl mit der Theorie von JakobsobnLask höchstens nur soviel erklärt werden, dass im Zentralnervensystem verschiedene Arten gekreuzter Fasern vorkommen, aber die Ursache der Kreuzung der spezifisch differenzierten Leitungsbahnen oder Fasersysteme im Zentralnervensystem bleibt in Dunkel gehüllt.

Drittens ist die Ansicht von Jakobsohn-Lask, dass die partielle Kreuzung der Nervenbahnen in der tierischen Entwicklung das Primäre gewesen sei und die totale sich an ganz wenigen Stellen aus besondern Unständen herausgebildet habe, auch nicht genug begründet. Der Hauptgrund dafür besteht nach ihm darin, dass die Nervenbahnen, wie schon erwähnt, ursprünglich aus einem diffusen Netz hervorgegangen seien. Diese Argumentation ist aber ebenso wenig begründet wie seine eben beschriebene Erklärungsart für die Kreuzung der Leitungsbahnen. Wie seine Hypothese die Tatsache, dass die aus mehreren Neuronen zusammengesezten Bahıen, -wenn auch Möglichkeiten mehrmaliger verschiedenartiger Kreuzungen vorliegen, -in der Tat sich meistens nur einmal kreuzen, nicht genügend zu erklären vermag, ebensowenig kann sie auch auseinandersetzen, ob die Kreuzung der Nervenbahnen primär partiell oder total sei, demn sie kam sich ja ebenso gut primär total wie partiell kreuzen, wenn die Nervenbahnen auch aus dem Netz entstanden wären.

Aus dem Gesagten geht hervor, dass man trotz allen Bemühungen der Vorgänger noch nicht in der Lage ist, die Kreuzung der Nervenbahnen im Zentralnervensystem zu ergründen. Im Folgenden will ich nun über die biologische Bedeutung derselben meine eigene Meinung äusserı.

Wie schon Spitzer, Jakobsohn-Lask u.a.m. hervorheben, ist die Kreuzung der Leitungsbahnen eine im ganzen Tierreich weitverbreitete Erscheinung, was gerade bezeugt, dass sie eine allgemeine biologische Bedeutung haben muss. Da es aber vom strieng wissenschaftlichen Standpunkt aus aus dem oben erwähnten Grunde heute noch gefährlich ist, die Frage, das ganze Tierreich von den Wirbellosen bis zu den Wirheltieren hindurch umfassend, zu erörtern, so beschränke ich mich in die- 
ser Abhandlung, um möglichst sachlich zu bleiben, auf das Wirbeltierreich und zwar auf das Zentralnervensystem desselben.

Nach meiner Auffassung besteht die biologische Bedeutung der Leitungsbahnen darin, die beiderseitigen Hälften des Zentralnervensystems, sowohl morphologisch als auch funktionell, miteinander zu einem Ganzen zu vereinigen, um der Gefahr einer Dissoziation derselben möglichst vorzubeugen.

Diese Rolle kommt aber nicht nur den gekreuzten Fasern der Leitungsbahnen, sondern auch den Kommissurenfasern zu. Die Kreuzung der Leitungsbahnen ist also, wemn man sie vom Standpunkt der eigentlichen Rolle derselben, die Erregungen von einem Abschnitt des Nervensystems zum anderen fortzuleiten, aus betrachtet, mehr eine Einrichtung von sekundärer Bedeutung, welche neben der Hauptfunktion, nämlich der Reizleitung, gleichzeitig eine enge Verbindung der beiderseitigen Hälften des Zentralnervensystems und folglich des Körpers herstellt. Mit andern Worten stellt die Kreuzung der Leitungsbahnen für die Reizleitung selbst keinen unentbehrlichen Faktor dar, sondern sie gewinnt erst für die innige Verbindung der beiderseitigen Hälften des Zentralnervensystems eine grosse Bedeutung, welche aber auch den Kommissurenfasern z.T. gemeinsam zukommt. Um dies klar zu machen, müssen wir den Faserarten und der Zusammensetzung der Leitungsbahnen Erwähnung tun.

Im Zentralnervensystem finden sich, wie bekannt, principiell dreierlei Arten Fasern, nämlich die Assoziations-, die Kommissuren- und die Bahnfasern. Biologisch betrachtet, funktionieren alle diese Fasern nicht voneinander unabhängig, sondern sie müssen alle unter Arbeitsteilung einem gemeinsamen Zweck, verschiedene Lebensfunktionen harmonisch zu regulieren, dienen.

Die Assoziationsfasern verbinden die verschiedenen Teile innerhalb eines bestimmten Abschnittes einer Gehirn- oder Rückenmarkshälfte, die Kommissurenfasern dagegen die der beiden Hirn- und Rückenmarkshälften, während die Bahnfasern verschiedene, von einander weit entfernte Hirn- und Rückenmarksabschnitte miteinander, sowie diese einerseits und die Körperperipherie anderseits in Verbindung setzen.

Von diesen drei Faserarten kreuzen sich die Kommissurenfasern stets vollkommen und die Bahnfasern in den meisten Fällen teilweise oder vollständig. Weil meines Erachtens die Kommissuren und Bahnfasern aus dem eben erwähnten Grunde keine principiell verschiedene Faserarten sind, so müssen die Kreuzungen dieser beiden Arten Fasern biologisch auch etwas Gemeinsames haben. 
Die Leitungsbahnen im Zentralnervensystem setzen sich aus einer bestimmten Anzahl Neuronen zusammen. Führe ich nur einige Beispiele an, so setzen sich die Pyramidenbahnen aus zwei Neuronen zusammen und kreuzen sich teilweise immer an den Fasern erster Ordnung. Die extrapyramidalen Bahnen bestehen immer wenigstens aus mehr als drei Neuronen, wenn man die ganze Strecke vom Ursprung bis zur peripheren quergestreiften Muskulatur in Betracht zieht. Der Tractus rubrospinalis setzt sich z.B. wenigstens aus drei oder sechs Neuronen zusammen, je nachdem das erste Neuron aus der Grosshirnrinde direkt zum Nucleus ruber gelangt bezw. im Thalamus endet. Im ersteren Fall entsprechen die grossen motorischen Zellen im Nucleus ruber den zweiten Neuronen, deren Achsenzylinder sich in der Forel'schen Haubenkreuzung kreuzen, und die Vorderhornzellen den dritten. Im letzteren Fall liegt das zweite Neuron im Thalamus, das dritte im Striatum, das vierte inı Pallidum, das fünfte, dessen Achsenzylinder sich kreuzt, im Nucleus ruber, und das sechste im Vorderhorn des Rückenmarks. In der Tat ist aber der Tractus rubrospinalis meistens aus mehr Neuronen zusammengesetzt als eben angegeben, weil sich zwischen den genannten Hauptneuronen, welche lange Achsenzylinder abgeben, noch sog. Schaltneurone einschieben können.

Die Bahuen für die Hautsensibilität setzen sich principiell aus drei Neuronen zusammen, von denen sich das zweite stets kreuzt. Dies gilt auch im grossen und ganzen für die Bahnen des Geschmacks- und Gehörsinnes und vielleicht noch für die des Geruchsimnes(?). Die Sehbahn setzt sich aus zwei Neuronen zusammen, wenn man von der Ganglienzellenschicht der Retina zählt, und kreuzt sich total oder partiell im ersten Neuron.

Durch die Kreuzung der Leitungsbahnen werden entferntere und durch die der Kommissurenfasern nähere Teile der beiden Hälften des Zentralnervensystems unmittelbar verbunden, sodass diese unter stetiger Mitwirkung harmonisch arbeiten und der Gefahr der Dissociation entgehen. Dies ist gewiss für das Lebewesen eine dankbare Einrichtung. Wie unangenehm wäre es, wenn gekreuzte Fasern nicht existierten und sich die beiden Hälften des Gehirns und des Rückenmarks leicht dissocieren würden!

Man könnte wohl den Einwand erheben, dass die Leitungsbahnen sich nicht $z u$ kreuzen brauchten, wenn ihre Kreuzungen einfach in der innigen Verbindung der beiden Hälften des Zentralnervensystems und der Vorbeugung der Dissociation derselben beständen, da im Zentralnervensystem schon überall die die genamnte Funktion übernehmenden 
Kommissurenfasern vorhanden sind. Dem ist aber nicht so, denn durch die Kommissurenfasern werden, wie schon gesagt, nur nähere Stellen innerhalb eines bestimmten Abschnittes des Zentralnervensystems verbunden, aber nicht von einander entfernte Teile desselben. Die weit eutfernten Stellen im Zentralnervensystem miteinander und diese einerseits und die Körperperipherie anderseits können nur durch Bahnfasern verbunden werden. Man sieht also klar, dass die Kreuzung der Leitungsbahnen auch zum Zweck der kommissurellen Funktion nicht überflüssig, sondern sogar unentbehrlich ist.

Besonders interessant ist der Grad der Kreuzung der Leitungsbahnen, nämlich ob sie eine totale oder eine partielle sei. Auf die Frage, ob die totale oder die partielle Kreuzung das Primäre sei, kann auch ich nicht bestimmt antworten, da unsere Kenntnis in dieser Hinsicht noch recht dürftig ist. Ich bin aber eher zur Amnahme geneigt, dass die totale Kreuzung die primäre und die partielle die sekundäre sei. Die vergleichend-anatomische experimentelle Untersuchung der Rückenmarkspyramide vom Verfasser spricht auch dafür, indem die Rückenmarkspyramide bei niederen oder bei denjenigen Wirbeltieren, bei welchen die motorische Funktion noch nicht genug differenziert ist, eine vollkommene Kreuzung, dagegen bei höheren oder bei denjenigen Wirbeltieren, bei denen die motorische Funktion stark differenziert ist, eine partielle Kreuzung erfährt. Hiermit meine ich aber nicht, dass es im Zentralnervensystem solche Leitungsbahnen nicht gebe, welche sich gerade umgekehrt zuerst partiell und sekundär total kreuzen, und ferner, dass auch ungekreazte Bahnen nicht existieren sollen. Es braucht wohl nicht besonders erläutert zu werden, dass die partielle Kreuzung der totalen gegenüber einen Vorzug hat, da die beiden Hälften des Gehirus oder des Rückenmarks bei ersterer weniger der Gefahr der Dissociation ausgesetzt ist als bei letzterer.

Das Verhältnis der gekreuzten zu den ungekreuzten Fasern bei den einzelnen Leitungsbahnen ist bis heute meistens noch nicht sicher ermittelt worden, aber der Hauptsache nach würden wohl erstere zahlreicher als letztere sein, was für die Tiere auch nützlicher ist als umgekehrt. Theoretisch betrachtet, wäre die ideale Kreuzungsart der Leitungsbahnen eine vollkommene Semidekussation, in welcher eine Hälfte der Fasern gekreuzt und die andere ungekreuzt verläuft. $\mathrm{Ob}$ dies wirklich der phylogenetischen Entwicklung der Tiere parallel geht, darüber lässt sich heute noch nichts Bestimmtes sagen, doch findet man wohl schon im phylogenetischen Verhalten des N. opticus, der Pyramidenbahn usw. Andeutungen dazu. Die vergleichend-anatomische 
Forschung einzelner Bahnen ist in dieser Hinsicht recht wüuschenswert.

Auch die Tatsache, dass sich die Kreuzung der Leitungsbahnen, jede für sich betrachtet, auf eine bestimmte Stelle des Zentralnervensystems beschränkt, aber als Ganzes betrachtet, sich auf das ganze Gebiet derselben erstreckt, ist für die Tiere sehr nützlich, indem dadurch solche Gefahren, dass sich die durch Schädigung eimes bestimmten Teiles des Zentralnervensystems hervorgerufenen Störungen auf einer Hälfte des Körpers konzentrieren und diese sozusagen invalid machen, zum grössten Teile vermieden werden.

Zum Schluss möchte ich durch ein konkretes Beispiel zeigen, auf welche Weise die Kreuzung der Leitungsbahnen den Lebewesen nützlicher ist und der Dissociation der beiden Hälften des Zentralnervensystems und folglich des Körpers vorbeugend wirkt. Als Beispiel wähle ich hier die sog. Brown-Séquardsche Halbseitenläsion des Rückenmarks. Hier fiuden sich, wie bekannt, auf der Seite der Läsion infolge Durchtremnung der motorischen Bahnen und zwar vor allem der gekreuzten Pyramidenseitenstranghahn sowie der vielen extrapyramidal-motorischen Bahnen motorische Lähmungen und infolge Läsion der aufsteigenden Hinterstrang- und Kleinhirnseitenstrangbahnen Störungen der Tiefensensibilität, auf der gekreuzten Seite infolge Läsion des gekreuzt verlaufenden Tractus spinothalamicus Störungen der Schmerz- -und Temperaturempfindung. Diese Verteilung der Störungen auf die beiden Seiten des Körpers, was biologisch für das Lebewesen viel nützlicher ist, als dass die Störungen auf einer Seite bleiben würd€n, käme nicht vor, wemn die Leitungsbahnen alle ungekreuzt verliefen, oder weun sich diese, obgleich gekreuzt, ganz konzentriert an einer derselben Stelle kreuzten. In Wirklichkeit kreuzen sich aber die Leitungsbahneu, wie oben gesagt, meistens partiell oder total und zwar als Ganzes betrachtet, zerstreut auf der ganzen Strecke des Zentralnervensystems.

Ferner ist es leicht, zu vermuten, dass die ungekreuzten Pyramidenseitenstrang- und Pyramidenvorderstrangbahnen der der Läsion gegenüberliegenden Rückenmarkshälfte, deren Fasern auch nach der allgemeinen Annahme im Rückenmark eine nachträgliche präterminale Kreuzung erfahren, uın die Vorderhornzellen der gekreuzten Seite zu endigen, mehr oder weniger zu der sog. Kompensation der motorischen Störung beitragen, welche aus der Läsion der Hauptpyraınide, d.h. der gekreuzten Pyramidenseitenstranghahn der geschädigten Seite herrührt.

Aus dem Gesagten glaube ich also, dass meine Betrachtungsart bezüglich der hiologischen Bedeutung der Kreuzung der Leitungsbahnen 
Zur biologischen Bedeutung der Kreuzung der Leitungsbahnen im Zentrelnervensystem. 329

klarer und eindeutiger ist, als irgend eine andere, bisher aufgestellte Hypothese.

\section{Literaturverzeichnis.}

1. Cajal, R. y, Die Struktur des Chiasma opticum nebst einer allgemeinen Theorie der Kreuzung der Nervenbahnen. Úbersetzt von J. Bresler. 1899. Cit. n. Jakobsohn-I.ask.

2. Flechsig, P., Die Jeitungsbahnen im Gehirn und Rückenmark. J.eipzig, 1876.

3. Hirasawa, K., Über das extrapyramidale motorische System. Jûzenkai Z. Bd. 38, 1933. (Japanisch.)

4. Jakobsohn-Lask, L., Die Kreuzung der Nervenbahnen und die bilaterale Symmetrie des tierischen Körpers. Berlin, 1924.

5. —_ Warum kreuzen sich die Leitungsbahnen im Zentralnervensystem ? Zeits. f. ges. Neur. u. Psychiatr. Bd. 112, 1923.

6. Kappers, Ariens C. U., On Neurobiotaxis. A psychical law in the structure of the nervous system. Acta psychiatrica et neurologica. Bd. 2, 1927.

7. Kato, K., Vergleichend-anatomische experimentelle Untersuchung der corticospinalen Bahnen. Hokuetsu Igaku Z. 1934. (Japanisch.)

8. Miskolczy, D., Warum kreuzen sich die zentralen Bahnen des Nervensystems? Zeits. f. d. ges. Anat., Abt. 1 :Zeits. f. Anat. u. Entwicklungsgesch. Bd. 81, 1926.

9. Radel, E., Neue Lehre von Nervenbahnen. J.eipzig, 1910.

10. Spitzer, A., Über die Kreuzung der zentralen Nervenbahnen und ihre Beziehungen zur Phylogenese des Wirbeltierkörpers. 1910.

11. W undt, W., Grundzüge der physiologischen Psychologie. 6. Aufl. 1908. 